DR DENNY L COTTLE (Orcid ID : 0000-0001-5047-6515)

Article type : Research Letter

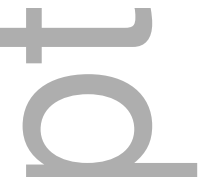

\title{
BJD Research Letter: A profile of lipid dysregulation in Harlequin Ichthyosis
}

Word count: 849 (Body text)/800 Figures: 1/1 References: 10/10

S. C. I. Ip ${ }^{1,2 *}$, D. L. Cottle ${ }^{1,2^{* *}}$, L. K. Jones ${ }^{1,2}$, J. M. Weir ${ }^{3}$, D. P. Kelsell ${ }^{4}$, E. A. O'Toole ${ }^{4}$, P. J. Meikle ${ }^{3}$ and I. M. Smyth ${ }^{1,2,5 \#}$.

1) Monash Biomedicine Discovery Institute, 23 Innovation Walk, Monash University, Wellington Rd, Clayton 3800, Victoria, Australia

2) Department of Biochemistry and Molecular Biology, Monash University, Wellington Road, Clayton 3800, Victoria, Australia.

3) Baker IDI Heart and Diabetes Institute, 75 Commercial Road, Melbourne 3004, Victoria, Australia.

4) Centre for Cell Biology and Cutaneous Research, Blizard Institute, Barts and the London School of Medicine and Dentistry, Queen Mary University of London, Whitechapel, London E1 2AT, UK.

5) Department of Anatomy and Developmental Biology, Monash University, Wellington Road, Clayton 3800, Victoria, Australia.

*these authors contributed equally to this study

\section{\#Corresponding authors}
A/Prof Ian M. Smyth
Dr Denny L. Cottle
Tel: +61 $39902-9119$
Tel: +61 3 9902-9294
Fax: +61 $39905-2500$
Email: ian.smyth@monash.edu
Email: denny.cottle@monash.edu

This is the author manuscript accepted for publication and has undergone full peer review but has not been through the copyediting, typesetting, pagination and proofreading process, which may lead to differences between this version and the Version of Record. Please cite this article as doi: $\underline{10.1111 / \text { bjd. } 15642}$

This article is protected by copyright. All rights reserved 


\section{Statement of funding}

This work was funded by a National Health and Medical Research Council (NHMRC Australia) project grant (APP1020281). SCII received an Australian Post-graduate Award. IMS was supported from a NHMRC R. Douglas Wright Fellowship, an Australian Research Council Future Fellowship and a Monash University Fellowship and currently by a NHMRC Senior Research fellowship.

Conflict of Interest Statement: The authors declare no competing interests.

Dear Editor,

Harlequin Ichythosis (HI) is a severe skin disease caused by mutations in the lipid transport protein, ABCA12. This disrupts extracellular lipid deposition; limiting barrier function and resulting in the differentiation of a thickened, restrictive epidermis. This phenotype is at least partially recapitulated in mouse models of disease. While patients ${ }^{1}$ (and HI mice ${ }^{2}$ ) exhibit a partial phenotypic correction after birth, they still require a life-long regime of frequent bathing, mechanical desquamation and emollient application.

The ceramides are the major lipid species in the intercellular lipid lamellae of the stratum corneum. These lamellae are established and maintained by lamellar bodies (LB's) which release their lipid cargo from terminally differentiating keratinocytes. While ABCA12 is required for the normal loading of LBs with glucosylceramide, the broader impacts of its dysfunction on lipid homeostasis have not been assessed. Ceramide can be produced as an intercellular deglucosylation product of glucosylceramide ${ }^{3}$, which is itself synthesised from dihydroceramide. It can also be produced by sphingomyelin conversion ${ }^{3}$. A further ceramide derivative, acylceramide, is predominantly formed from glucosylceramides in a process requiring the acyl chains of triacylglycerol and diacylglycerol ${ }^{4,5}$, although alternative pathways for the production of this species are possible ${ }^{4}$. Loss of waterproofing acylceramides (and accumulation of their glucosylceramide precursors) has been proposed as an underlying mechanism for the development of barrier defects in $\mathrm{HI}^{2,6,7}$.

Despite advances in our understanding of cutaneous lipid composition and the changes associated with loss of ABCA12, studies of lipid dysregulation have been limited to a small number of lipid species. In addition there have been no exhaustive comparative studies of patient skin versus mouse models or of changes associated with the transition to postnatal life. To address this we performed mass spectroscopy (MS) of >180 lipid species ${ }^{8}$ in epidermal peels from fetal (E17.5) and grafted (14 days post graft) skin from the el12 mouse model of $\mathrm{HI}^{9}$ and from $\mathrm{HI}$ patient (and control) epidermal scrapes from sites not treated with moisturisers or emollients. Our analysis This article is protected by copyright. All rights reserved 
includes an extensive range of other lipids known to be important for epidermal biology. Here we report those that were significantly altered in our samples; providing a complete analysis as supporting data.

We firstly profiled lipid disequilibrium in HI patients and grafted HI mouse skin. We observed increases in ceramide, glucosylceramide, dihydroceramide and acylceramide (Cer[EOS]) species compared to control samples (Fig. 1a-d). We also observed an increase in acylglucosylceramides (GlcCer[EOS]) in mouse grafts (Fig. 1e) although these species were not detected in human samples (likely due to limitations of epidermal scrapes compared to whole epidermis). The acquisition of acylceramides in human samples correlated with a general depletion of many diacylglycerol and triacylglycerol species (Fig. 1f,g) and while statistical significant was not achieved in murine graft samples, diacylglycerol and triacylglycerol did show a similar downward trend which paralleled significant increases in acylceramides. The total sphingomyelin pool was upregulated in human samples, however the individual moieties often exhibited significant yet opposite regulation between human and mouse species (Fig. 1h). We next profiled lipid changes associated with the transition of skin to a postnatal environment and found significant increases in the levels of ceramides, glucosylceramides, dihydroceramides, acylceramides and acylglucosylceramides in postnatal skin versus fetal skin (Fig. 1a-e). These observations fit with a broader picture that lipid dysregulation is exacerbated during the transition from an aqueous to a gaseous environment at birth which presumably occurs as part of the general response of the organ to a loss of barrier function.

Several other aspects of the study are worth noting. We have previously reported that ABCA12 plays a role in cholesterol efflux ${ }^{9}$ and observed this trend again here in mouse skin, but surprisingly such lipids were often downregulated in human HI patients (Fig. 1i). In addition, the overall results demonstrate that mouse models of postnatal HI closely parallel the differences in lipid homeostasis observed in HI patients when examining total lipids of a given family although may be divergent when comparing individual moieties within a lipid group. There were also a number of specific lipid species whose variation, while robust, contrasted to other members of the same lipid family, such as sphingomyelin species. The significance of these changes is unclear, but they may point to specific roles for these lipids in disease development or particular synthesis pathways. At a mechanistic level our study suggests that the postnatal upregulation of acylceramides observed in mice and humans occurs via a dihydroceramide and sphingomyelinlinked ceramide synthesis pathway (Fig. 1j) which concurs with a recent study that suggested ceramides in HI skin derive principally from sphingomyelin ${ }^{7}$. They further indicate that mouse models of $\mathrm{HI}$ represent a promising platform upon which to examine disease pathogenesis and act as a system in which to profile adaption of the organ to a new environment at birth. Indeed, our 
results correlate with recent observations demonstrating that accumulation of intracellular ceramide can cause ichthyosis ${ }^{10}$, suggesting that the ichthyosis phenotype is not purely due to loss of waterproofing extracellular lipids. Importantly, our studies also establish a baseline upon which to assess correction of disease in preclinical trials of HI therapies aimed at normalising lipid levels or correcting defects in keratinocyte differentiation/function.

\section{Acknowledgements:}

We thank Dr. Aidan Sudbury (Monash University) for expert advice on statistical methods and the patients and volunteers who provided samples. We also thank the Monash Histology Platform for their services.

\section{Statement of Author Contributions:}

SCII, DLC, LKJ and IMS performed experiments, DPK and EAO'T provided human HI epidermal scrapes. PJM and JMW ran the lipid MS. DLC analysed the data and DLC and IMS wrote the paper. IMS conceived and directed the project.

\section{Reference List:}

1 Rajpopat S, Moss C, Mellerio J et al. Harlequin ichthyosis: a review of clinical and molecular findings in 45 cases. Arch Dermatol 2011; 147: 681-6.

2 Yanagi T, Akiyama M, Nishihara $\mathrm{H}$ et al. Self-improvement of keratinocyte differentiation defects during skin maturation in ABCA12-deficient harlequin ichthyosis model mice. Am J Pathol 2010; 177: 106-18.

3 Feingold KR. Thematic review series: skin lipids. The role of epidermal lipids in cutaneous permeability barrier homeostasis. J Lipid Res 2007; 48: 2531-46.

4 Takagi Y, Nakagawa H, Matsuo N et al. Biosynthesis of acylceramide in murine epidermis: characterization by inhibition of glucosylation and deglucosylation, and by substrate specificity. J Invest Dermatol 2004; 122: 722-9.

5 Stone SJ, Myers HM, Watkins SM et al. Lipopenia and skin barrier abnormalities in DGAT2-deficient mice. J Biol Chem 2004; 279: 11767-76.

6 Zuo Y, Zhuang DZ, Han R et al. ABCA12 maintains the epidermal lipid permeability barrier by facilitating formation of ceramide linoleic esters. J Biol Chem 2008; 283: 36624-35.

7 Haller JF, Cavallaro P, Hernandez NJ et al. Endogenous beta-glucocerebrosidase activity in Abca12-/-epidermis elevates ceramide levels after topical lipid application but does not restore barrier function. J Lipid Res 2014; 55: 493-503. 
Weir JM, Wong G, Barlow CK et al. Plasma lipid profiling in a large population-based cohort. J Lipid Res 2013; 54: 2898-908.

9 Smyth I, Hacking DF, Hilton AA et al. A mouse model of harlequin ichthyosis delineates a key role for Abca12 in lipid homeostasis. PLoS Genet 2008; 4: e1000192.

Amen N, Mathow D, Rabionet M et al. Differentiation of epidermal keratinocytes is dependent on glucosylceramide:ceramide processing. Hum Mol Genet 2013; 22: 4164-79.

\section{Figure Legends:}

Figure 1: Lipid profiling in HI epidermis. a-i) Lipid analysis of E17.5 embryonic epidermis, embryonic skin grafted onto Nude mice and analysed 14 days post graft (14dpg) and human epidermal scrapes. Lipids were internally normalised to total PC. Lipid values obtained were then expressed as a fold change relative to the average value obtained from type-matched Control WT skins (arbitrary defined as 1). Lipid values obtained from embryonic skins were expressed relative to E17.5 WT embryonic skins, grafted skins were expressed relative to 14dpg WT grafted skins, and human epidermal scrapes expressed relative to WT human samples. Lipid data are represented as heatmaps generated with CIMminer (http://discover.nci.nih.gov/cimminer/home.do) using the quantile method. Blank spaces within heatmaps indicate lipid species were not detected. Significance for embryonic skin and grafts was determined by Student's T-test where asterisks indicate $* p<0.05, * * p<0.01, * * * p<0.005$. Statistical significance for human scapes was determined using a Mann Whitney U-test where $* \mathrm{p}<0.036$. Full analysis is available in supplementary material. j) A model for barrier lipid trafficking/conversion in normal skin. Predominant defect in HI indicated; proposed alternate pathways are indicated by grey arrow.

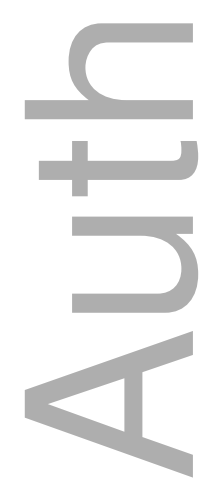

This article is protected by copyright. All rights reserved 
(a) Ceramides

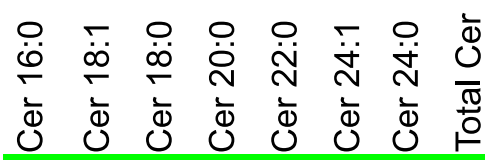

Controls (def. as 1)

E17.5 el12/el12

14dpg el12/e/12

human $H /$ scrape

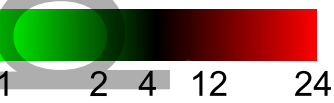

-

(d) Acylceramides

Controls (def. as 1)

E17.5 el12/e/12

14dpg el12/e/12

human $H I$ scrape

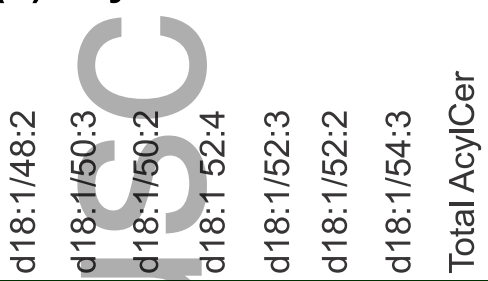

$$
\text { ) }
$$

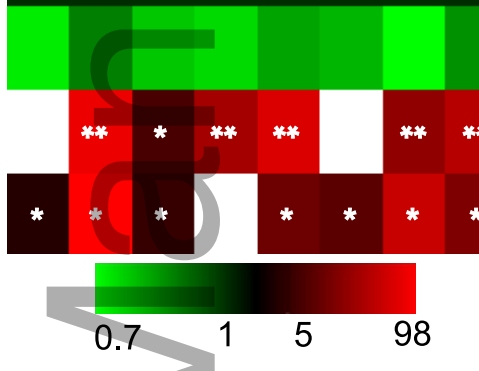

(g) Triacylglycerol

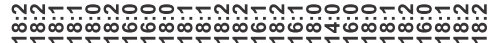

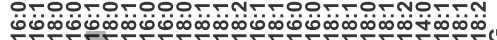

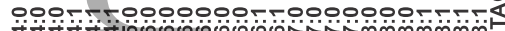

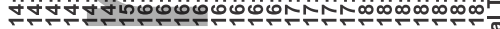
0000000000000000000000000

Controls (def. as 1)

E17.5 el12/el12

14dpg e/12/e/12

human $H$ I scrape
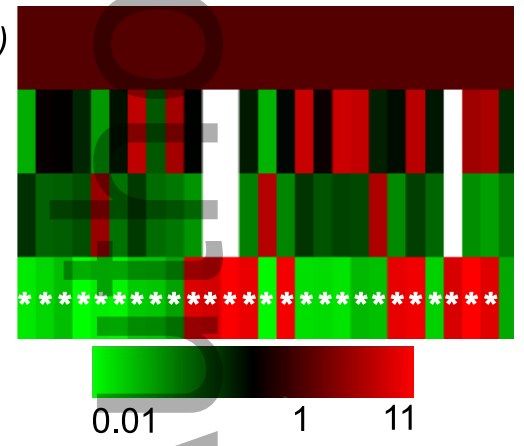

(j) Lipid Pathway

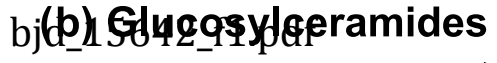

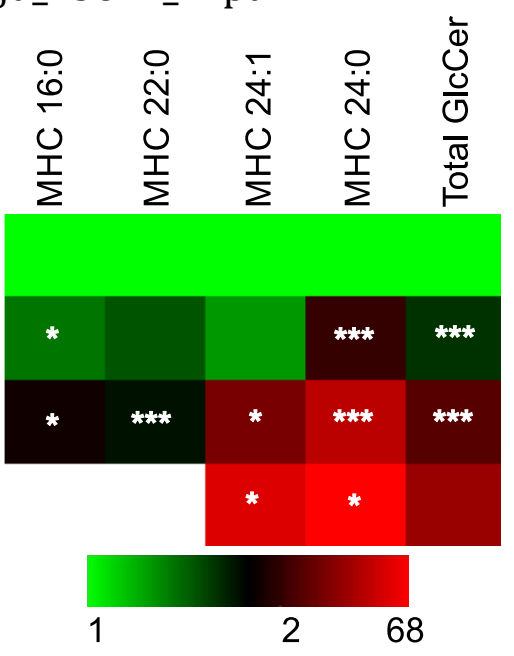

(e) Acylglucosylceramides
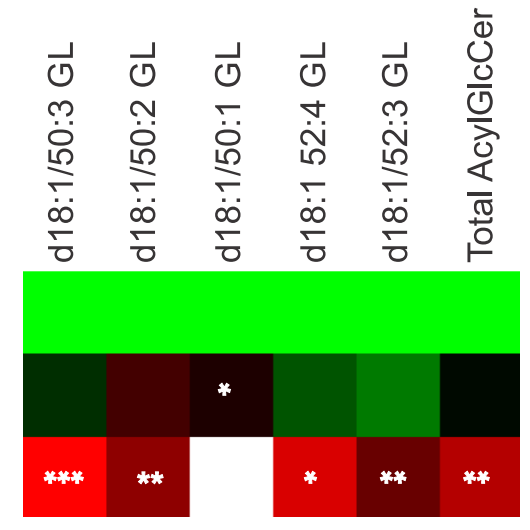

(Not detected)

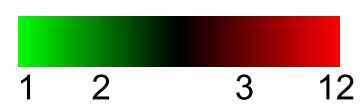

(h) Sphingomyelin
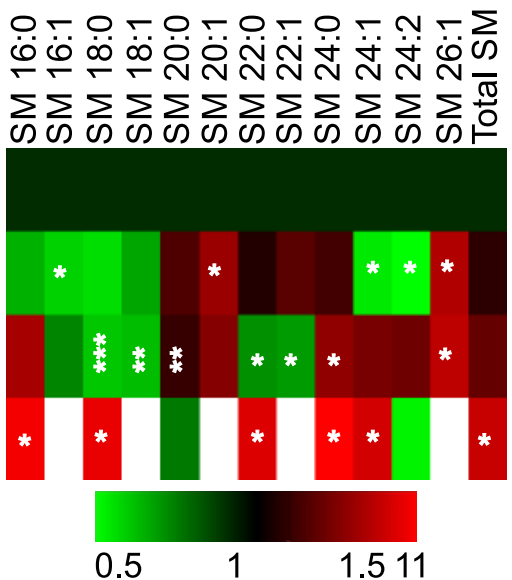

(c) Dihydroceramides

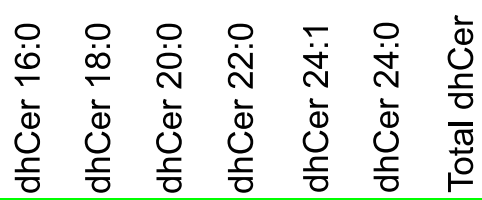
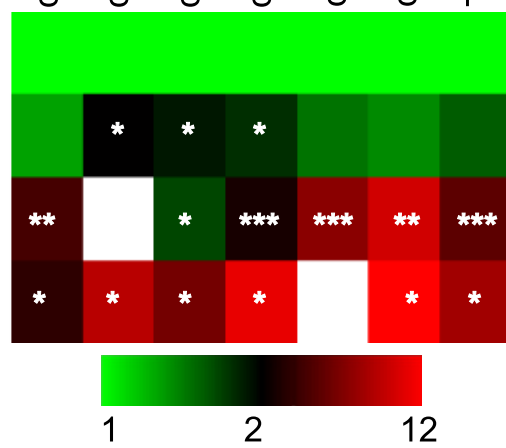

\section{(f) Diacylglycerol}

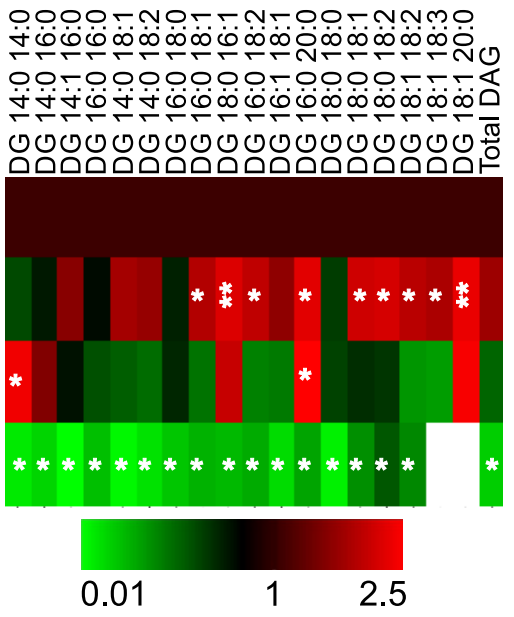

\section{(i) Cholesterol \& Esters}

I

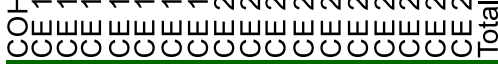

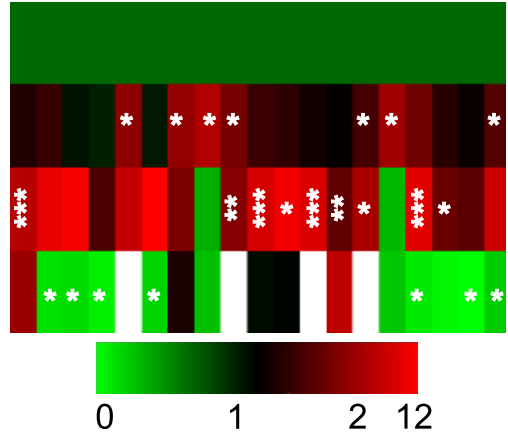

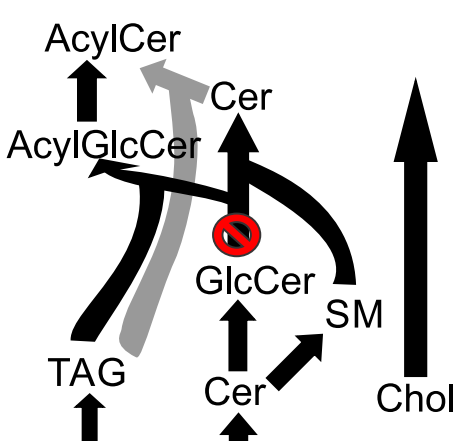

Extracellular

This article is protected by copyidiget. All $\mathrm{r}$ fhts reserved Intracellular dhCer 


\section{University Library}

\section{- M M N E R VA A gateway to Melbourne's research publications}

Minerva Access is the Institutional Repository of The University of Melbourne

Author/s:

Ip, SCl;Cottle, DL;Jones, LK;Weir, JM;Kelsell, DP;O'Toole, EA;Meikle, PJ;Smyth, IM

Title:

A profile of lipid dysregulation in harlequin ichthyosis

Date:

2017-11-01

Citation:

Ip, S. C. I., Cottle, D. L., Jones, L. K., Weir, J. M., Kelsell, D. P., O'Toole, E. A., Meikle, P. J. \& Smyth, I. M. (2017). A profile of lipid dysregulation in harlequin ichthyosis. BRITISH JOURNAL OF DERMATOLOGY, 177 (5), pp.E217-E219. https://doi.org/10.1111/bjd. 15642.

Persistent Link:

http://hdl.handle.net/11343/293746 